\title{
Three-phase contact line shape and eccentricity effect of anisotropic wetting on hydrophobic surfaces
}

\author{
Navid Kashaninejad, ${ }^{a}$ Nam-Trung Nguyen ${ }^{a}{ }^{a}$ and Weng Kong Chan ${ }^{a}$ \\ Received 25th August 2012, Accepted Xth XXXXXXXXX 20XX \\ ${ }_{5}$ DOI: 10.1039/b000000x
}

This paper experimentally evaluates the combined effects of eccentricity, relative spacing, and viewing directions on the wetting conditions and the three-phase contact line shapes of hydrophobic surfaces patterned with discrete micropillars. Different techniques to depict the tortuosity of the contact line between the water droplet and microstructured surfaces are presented. First, square micropillars with 10 different values of normalized eccentricity, $\varepsilon^{*}$, and relative spacing, $D^{*}$, were fabricated using double casting replication technique. Subsequently, the contact angles were measured along different viewing angles by gradually rotating the sample from $0^{\circ}$ to $180^{\circ}$. The contact angle distribution was found as a periodic function of viewing angle whose period depends on the micropillar eccentricity. The results showed that anisotropy increases by increasing the micropillar eccentricity or decreasing the pillar

15 relative spacing. However, the effect of changing the micropillar eccentricity was much more pronounced. Micropillars with $\varepsilon^{*}=0.75$ and smaller $D^{*}$ showed maximum degrees of anisotropic wetting and droplet distortion corresponding to $7 \%$ and $15 \%$, respectively. Using the measured droplet aspect ratio, corrugated shapes of the three-phase contact line of the micropillars were also reconstructed. Finally, a simple yet effective semi-analytical model, based on Fourier series curve-fitting of the

20 experimental data, was developed to describe the equilibrium 3D shape of the droplet on anisotropic surfaces. Experimental and simulation results reveal that the degrees of anisotropic wetting and droplet distortion were directly proportional to the energy barriers of the system resulting from noncircular corrugated shape of the three-phase contact line. The obtained results may further shed light on the underlying mechanism influencing anisotropic wetting on micropatterned surfaces.

\section{${ }_{25}$ Introduction}

Controlling the wetting behavior of a droplet on a solid surface is of critical importance owing to its wide range of applications including surface coating, surface tension driven flow and liquid transport in emerging areas such as micro/nanofluidics, 30 biomedical devices, biomimetics, and digital microfluidics. Wettability is quantified by the contact angle which macroscopically manifests the intermolecular interactions at the three-phase contact line. In contrast to isotropic surfaces, the contact angle varies along different viewing angles on anisotropic 35 surfaces. Directional dependency of the contact angle known as anisotropic wetting is prevalent in nature such as allowing water droplet movement along the edge of rice, pandanus (screwpine) and bamboo leaves ${ }^{1-3}$, and rolling off (pinning) of drop along (against) the radial outward direction of the central axis of the 40 butterfly wings ${ }^{4}$. In general, asymmetry of either surface chemistry or surface morphology may lead to directional dependency of the contact angle. Accordingly, two methods can be devised to artificially fabricate anisotropic surfaces, namely one-dimensional (1D) and two/three-dimensional (2D/3D)

45 methods. In 1D approach, periodic planar regions of alternating hydrophobic and hydrophilic strips are formed using chemical coatings on macroscopically smooth surfaces. The wettability of these surfaces was analyzed experimentally, theoretically and numerically through the literature ${ }^{5-11}$. In 2D/3D approach, rough 50 surfaces with asymmetrical micro/nano protrusions are fabricated as a result of highly advanced micro/nano fabrication techniques. Because of its potential applications, attempts to identify the roles of key parameters influencing the anisotropic wetting are of great interest for the research community. Directional dependency of 55 the contact angle of different ranges of natural surfaces, such as rice leaf structures ${ }^{12}$, and other synthetic microstructures, including checkboard-patterned surfaces ${ }^{13}$, wrinkled surfaces ${ }^{14,15}$, ratchet-like topographical structures ${ }^{16}$, elliptical silicon arrays ${ }^{17}$ and stooped polymer nanohairs ${ }^{18}$, and tilted polymer nanorod 60 arrays [26], has been evaluated. Among many asymmetric microstructures, surfaces with periodic parallel grooves at different length scales, pitches, height, etc were extensively investigated in the literature ${ }^{19-34}$. Chen et al. ${ }^{20}$ reported a semianalytical and an experimental study of microgroove surfaces. ${ }_{65}$ Higher observable contact angle in the front view of the droplet compared to the side view was attributed to the squeezing and pinning of the droplet along the direction of the grooves and stretching across that direction. Neuhaus et al. ${ }^{34}$ investigated microstructured surfaces consisting of both groove and square 70 pillars with three different wetting conditions, namely hydrophobic, moderately hydrophilic and very hydrophilic. They 
found that on moderately hydrophilic groove substrate, a water droplet pinned to the structure and became elongated. The contact angle was considerably higher (up to $37^{\circ}$ ) along the viewing direction parallel to the groove as compared to that from the 5 perpendicular direction. In the case of square micropatterns, droplet shape deviated from being circular especially for the case of moderate hydrophilicity, and interestingly anisotropy was observed on those topographically symmetric structures. The contact angle was higher (around $4^{\circ}$ for the case of hydrophobic 10 and $9^{\circ}$ for the case of moderately hydrophilic conditions) along the edge as compared to the diagonal viewing direction of the square pillars. For both patterns in hydrophilic state, almost the same isotropic contact angle was reported. Long et al. ${ }^{29}$ systematically investigated the static and dynamic wetting 15 anisotropies of eight different translationally symmetric elongated ridges by gradually increasing the length of subsequent discrete ridge to finally form a continuous microridge structure. They reported that by splitting the continuous ridges into the discrete ones, the static and dynamic contact angle anisotropies 20 substantially decreased, but did not disappear.

Recently, we introduced another important geometrical parameter of the microstructured surfaces termed as eccentricity, $\varepsilon$, centreto-centre offset distance between successive columns of a square lattice of micropillars ${ }^{35}$. This parameter quantifies how much the 25 micropatterned surface deviates from being symmetric and is shown schematically in Fig. 1(a). As reported previously, increasing the values of the micropillar eccentricity not only makes the surface asymmetric but also increases the contact angle hysteresis ${ }^{35}$.The increase in contact angle hysteresis emphasises

30 the importance of the line tension, an additional term in the Gibbs free energy, as a result of the distortions of the three-phase contact line. The distorted nature of the contact line on eccentricity-induced asymmetric micropillars implies that contact angle may become different along different viewing angles, as 35 opposed to the non-distorted circular shapes of the contact line on isentropic surfaces. Thus, changing the eccentricity may lead to contact angle anisotropy. However, depicting the microscopic tortuosity of the three-phase contact line of a sessile water droplet on microstructured surfaces is a challenging problem. Alternative 40 methods to depict the contact line shapes of non-aqueous fluids use either the microscopic images of molten Woods after solidification $^{36}$ or SEM images of non-volatile organic liquids ${ }^{31}$. In this paper, we address this issue by systematically measuring the contact angles, widths, and heights at different viewing 45 directions, and schematically depict the water droplet three-phase contact lines using two-dimensional and three-dimensional methods. Additionally, the paper investigates the directional dependency of the contact angle as a function of eccentricity at different micropillar relative spacing. Wetting anisotropy of the 50 micropillar surfaces is quantified by introducing two new parameters, degree of anisotropic wetting and degree of anisotropic drop distortion. The effect of changing the micropillar eccentricity and spacing on these parameters is investigated and discussed. The results of the present paper can further elucidate 55 the effect of three-phase contact line of the micropillars, in terms of the micropillar eccentricity, and provide additional insight for further investigations to modify the existing wetting theories.

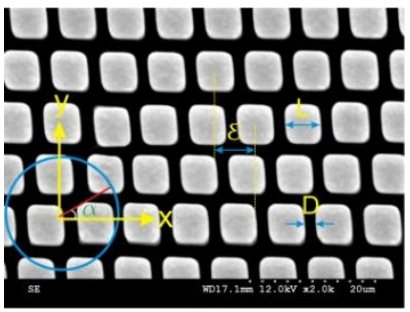

(a)

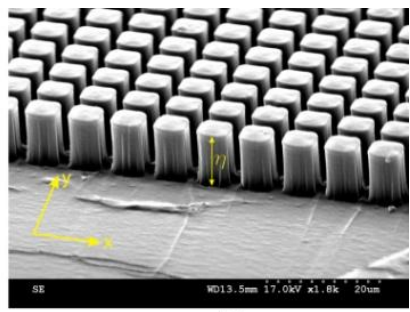

(b)
Fig. 1 Representative SEM images of the fabricated pillars: (a) Top view 60 of the micropillars with coordinates and geometrical parameters used in this study. (b) Isometric view of the micropillars, $\eta=10 \mu \mathrm{m}$.

\section{Experimental Section}

\section{Characterization of the Micropillars}

To evaluate the directional dependency, surfaces consisting 65 identical square micropillars with different values of spacing, $D$, and eccentricity $\varepsilon$ were designed and subsequently fabricated on polydimethylsiloxane (PDMS) substrates. To reduce the amount of experimental data, the geometrical parameters of the pillars including the micropillar spacing and eccentricity were 70 normalized with respect to the pillar pitch, $\lambda=L+D$. That is, $D^{*}=D / \lambda$ and $\varepsilon^{*}=\varepsilon / \lambda$, where $D^{*}$ is the relative pillar spacing and $\varepsilon^{*}$ is the normalized pillar eccentricity. The detailed geometrical parameters of the pillars are listed in Table 1 .

Table 1 Geometrical parameters of the fabricated PDMS square 75 micropillars $(\eta=10 \mu \mathrm{m}$ and $\lambda=8 \mu \mathrm{m})$

\begin{tabular}{ccccc}
\hline $\boldsymbol{L}(\boldsymbol{\mu} \boldsymbol{m})$ & $\boldsymbol{D}(\boldsymbol{\mu m})$ & $\boldsymbol{\varepsilon}(\boldsymbol{\mu m})$ & $\boldsymbol{D}^{*}$ & $\boldsymbol{\varepsilon}^{*}$ \\
\hline 6 & 2 & $0,2,4,6$ & 0.25 & $0,0.25,0.50,0.75$ \\
5.3 & 2.7 & & 0.33 & \\
\hline
\end{tabular}

\section{Micropillar Fabrication}

PDMS micropillars were formed using three-step double casting replication procedure. First, master mold micropillars were fabricated on silicon wafer using photolithography and deep 80 reactive ion etching (DRIE) techniques ${ }^{35}$. In the second step, microhole structures were transferred on PDMS following a standard micromolding method ${ }^{37}$. Finally, micropillars were formed on another PDMS substrate using double casting technique. To avoid irreversible bonding between two PDMS 85 substrates, the surface of the PDMS microholes template was treated with trichloromethylsilane (TCMS, Sigma Aldrich) as the releasing agent ${ }^{38}$ with the following procedure. First, oxygen plasma was used to render the PDMS microholes hydrophilic before the deposition of TCMS. Then, the PDMS slabs were 90 placed in a desiccator while $5 \mathrm{~mL}$ of TCMS was dripped in a Petri dish. The pressure of the chamber was reduced by pumping it for 90 minutes. Afterwards, the valve of the chamber connecting the pump was closed, and the pump was switched off. The total deposition process was conducted under a fume hood and the ${ }_{95}$ chamber was left overnight for the deposition to take place. After pouring another liquid PDMS on the TCMS-coated microhole substrate and solidifying it in an oven for 30 minutes at $85^{\circ} \mathrm{C}$, PDMS micropillars were successfully formed. The SEM images of these micropillar morphologies are shown in Fig. 1(a,b). 

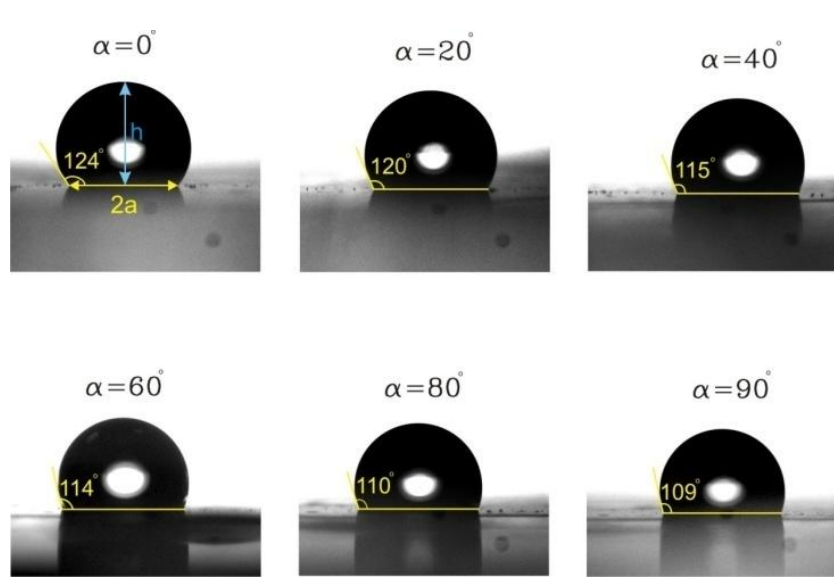

Fig. 2 Typical images of the contact angle anisotropy of the micropillar surfaces $\left(\mathrm{D}^{*}=0.25\right.$ and $\left.\varepsilon^{*}=0.75\right)$ of a single droplet by rotating the sample from $\left(0^{\circ}-90^{\circ}\right)$ in $20^{\circ}$ step increment.

\section{${ }_{5}$ Contact Angle Measurement}

Optical tensiometer (Theta Attension, BiolinScientific, Finland) was used to measure the contact angles and wettability conditions of the micropillar surfaces at the different viewing angles. The pixel error of the tensiometer is reported to be around 0.1 10 degrees.

\section{Drop Volume and Deposition Technique}

The degree of anisotropic wetting could be affected by the size of the droplet ${ }^{27}$. We needed to compromise between a large and a small volume of the droplet. A large droplet volume is more 15 stable but more prone to gravitational sagging. On the other hand, a small droplet volume is dominated by capillary pressure but evaporates quickly and may have a transient regime. Here, a water droplet of $2 \mu L$ was selected for the experiment. By denoting $\rho$ and $\gamma_{w}$ as the density and surface tension of water at 20 room temperature, and $r$ as the maximum droplet radius corresponding to the droplet volume of $2 \mu \mathrm{L}$, the Bond number is Bo $=\rho g r^{2} / \gamma_{w}=0.08$. This value of Bond number ensures that the influence of gravity is negligible compared to the surface tension. Having automatically pumped $2 \mu L$ volume of DI water 25 from the delivery syringe, the drop was detached at the minimum distance between the tip of the needle and the surface. The drop was placed gently on the sample without compressing it to keep the spherical shape and to minimize the perturbation and kinematic pressure. After a minute, the image of the drop was 30 captured and further analyzed using the built-in image analyzer of the tensiometer.

\section{Methodology and Statistical Error}

To evaluate the contact angle at each viewing direction systematically, each PMDS substrate consisting of four different 35 micropatterned surfaces was attached to a rotary stage (Edmund Optics Inc.) along the $y$ axis shown in the Fig. 1(a). The corresponding angle on the rotary stage was fixed to $90^{\circ}$ as the reference point. With the employed rotary stage, the sample can be rotated $360^{\circ}$ with $\pm 5^{\circ}$ fine rotations.

40 Moreover, each measurement was repeated at least 6 times at different locations of the sample (at each specific $D^{*}, \varepsilon^{*}$ and $\alpha$ ) to ensure the repeatability of the data and the average value of the contact angle was found at $95 \%$ confidence interval.
Corresponding statistical errors of the static contact angles were 45 less than $\pm 4^{\circ}$. Further error propagation and uncertainty analyses of the experimentally obtained parameters are presented in the Appendix.

Fig. 2 depicts a typical measurement of contact angle anisotropy of a single droplet with counterclockwise (CCW) $90^{\circ}$ rotation of 50 the sample $\left(D^{*}=0.25\right.$ and $\left.\varepsilon^{*}=0.75\right)$. This figure shows clearly the anisotropy of the fabricated surface, as the contact angle differs greatly at different viewing directions. Along the $\mathrm{x}$-axis $\left(\alpha=0^{\circ}\right.$ ) the contact angle is more than $124^{\circ}$ and as the sample rotates $\mathrm{CCW}$, it starts to decrease gradually, and the minimum 55 occurs along the orthogonal direction with a contact angle of less than $109^{\circ}$.

\section{Results and Discussions}

\section{Raw Experimental Data}
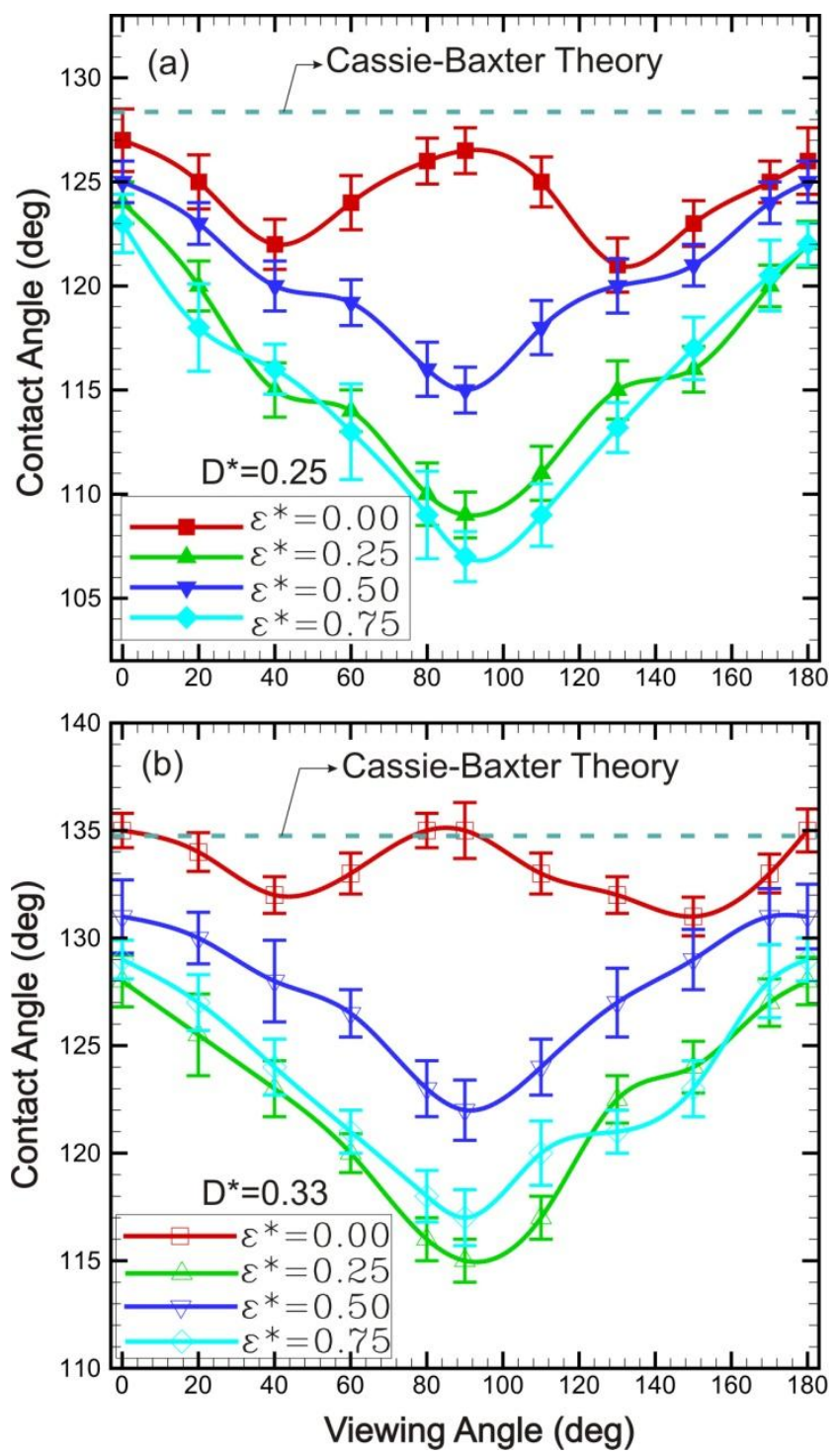

Fig. 3 Experimental contact angle $\theta$ versus viewing angle $\boldsymbol{\alpha}$ at different values of normalized eccentricity $\varepsilon^{*}$ and relative pillar spacing $D^{*}$. (a) $D^{*}=0.25$. (b) $D^{*}=0.33$. 
The average values of the contact angle on the micropillar surfaces of different $D^{*}$ and $\varepsilon^{*}$ as a function of viewing angles are shown in Fig. 3. In addition, the experimental values of the contact angle are also compared with the theoretical equation 5 proposed by Cassie and Baxter, $\theta_{C B},{ }^{39}$ :

$$
\cos \theta_{C B}=\phi_{s} \cos \theta_{\text {smooth }}+\left(1-\phi_{s}\right) \cos \pi
$$

where $\theta_{\text {smooth }}$ and $\pi$ are contact angles on the smooth solid and gas portions, respectively, and $\phi_{S}$ is the solid area fraction of the micropillars. The contact angle on smooth PDMS was measured 10 as $\theta_{\text {smooth }}=109^{\circ}$. The experimental contact angles on samples with zero eccentricities are in good agreement with Cassie-Baxter equation. However, the results indicate that this equation can only predict the maximum achievable contact angle irrespective of the micropillar eccentricities.

${ }_{15}$ Generally, the results show a periodicity trend for contact angle when the viewing angle gradually varies up to $180^{\circ}$. The period and amplitude (corresponding to the degree of contact angle anisotropy) of the samples are found to be a function of both micropillars normalized eccentricity and relative spacing. Most 20 importantly, the amplitude is larger for the case of lower pillar spacing, where the degree of hydrophobicity is lower. This implies that contact angle anisotropy becomes more important as the hydrophobicity decreases. Specifically, the results indicate that even on micropillars with zero eccentricity, the contact angle 25 is not isotropic. In this case, contact angles measured along the sides of the pillars $\left(\alpha=0^{\circ}, 90^{\circ}\right)$ are the same but decrease as viewing angle approaches the diagonal direction $\left(\alpha=45^{\circ}, 135^{\circ}\right)$. This is in qualitative agreement with the results reported by Neuhaus et al. $^{34}$, and can be related to the different geometrical 30 features. Thus, the droplet width at the three-phase contact line along the side is not equal to the one obtained along the diagonal direction. In that case, the droplet contact line deviates from the perfect circular shape.

By increasing the pillar normalized eccentricities to nonzero 35 values, the period of the data increases to $180^{\circ}$. That means, for the pillars with nonzero eccentricities, the maximum contact angle occurs along the $\mathrm{x}$-axis shown in Fig. 1(a). As the sample rotates, the contact angle decreases down to its minimum value along the perpendicular direction, i.e. y-axis in Fig. 1(a). 40 Additionally, the results indicate that along a fixed viewing angle, contact angle decreases by increasing the eccentricity, which is in good agreement with our results in a previous publication ${ }^{35}$.

\section{Degree of Anisotropic Wetting}

To further quantify the contact angle anisotropy, the degree of 45 anisotropic wetting, $\delta$, can be defined as:

$$
\delta=\frac{\theta(\alpha)_{\max }-\theta(\alpha)_{\min }}{\theta(\alpha)_{\max }+\theta(\alpha)_{\min }} \times 100 \%
$$

where $\theta(\alpha)_{\max }$ and $\theta(\alpha)_{\min }$ are maximum and minimum contact angles on the same micropillar surface obtained at different viewing directions. According to the experimental data, degree of 50 anisotropic wetting $\delta$ can be found as a function of normalized eccentricity $\varepsilon^{*}$ of the micropillars, Fig. 4.

The results suggest that the degree of anisotropic wetting of a surface can be tailored by changing both micropillar normalized eccentricity and relative spacing. As shown in Fig. 4, for the case

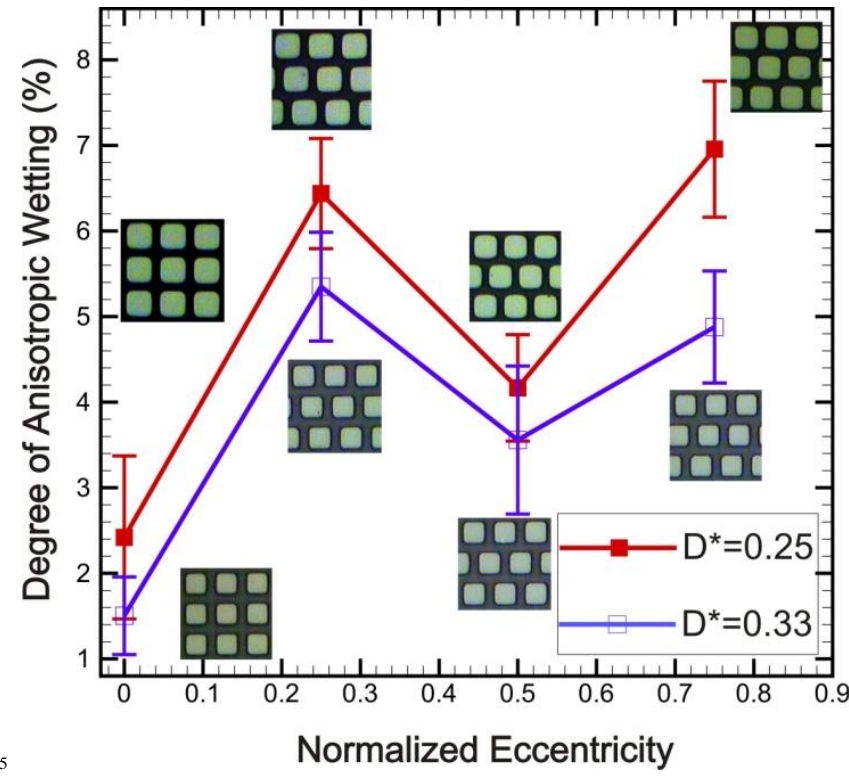

Fig. 4 Percentage of anisotropic wetting $\delta$ versus normalized eccentricity $\varepsilon^{*}$ at different values of relative pillar spacing $D^{*}$.

of lower pillar relative spacing, increasing the pillar eccentricity also increases the degree of anisotropic wetting. This can be 60 attributed to the increasing of the anisotropic features of micropillars by increasing the eccentricity.

Moreover, Fig. 4 indicates that the degree of contact angle anisotropy decreases by increasing the relative pillar spacing. For instance, on the surface with $D^{*}=0.25$ and $\varepsilon^{*}=0.75$, the ${ }_{65}$ degree of anisotropic wetting is as high as $7 \%$ while it falls below $5 \%$ by increasing the relative spacing to 0.33 . It was observed that as the relative pillar spacing increases, the degree of hydrophobicity also increases ${ }^{35}$. Therefore, the degree of contact angle anisotropy is inversely proportional to the degree of 70 hydrophobicity. This result suggests that for anisotropic but highly hydrophobic micropatterned surfaces, directional dependency of contact angle is less pronounced. This finding is also in agreement with the isotropic contact angle on the microstructures of lotus leaf ${ }^{40}$.

\section{${ }_{75}$ Degree of Anisotropic Droplet Distortion}

In the present paper, at each viewing angle, the average drop width, $2 a$, and the drop height $h$ were measured from the recorded images of the droplet, with the bias error of $\pm 0.0005 \mathrm{~mm}$, after proper calibration. Accordingly, the droplet 80 aspect ratio, $a^{*}=a / h$, was determined. Subsequently, similar to the degree of wetting anisotropy, we can define the degree of the anisotropic droplet distortion $\zeta$ as follows:

$$
\zeta=\frac{a^{*}(\alpha)_{\max }-a^{*}(\alpha)_{\min }}{a^{*}(\alpha)_{\max }+a^{*}(\alpha)_{\min }} \times 100 \%
$$

85 where $a^{*}(\alpha)_{\max }$ and $a^{*}(\alpha)_{\max }$ are the maximum and minimum droplet aspect ratios obtained at the different viewing angles $\alpha$ on the same micropillar surface.

The corresponding values of the degree of anisotropic droplet distortion $\zeta$ are plotted as a function of the normalized 90 eccentricity $\varepsilon^{*}$ in Fig. 5. The trend of increasing anisotropic drop distortion $\zeta$ is favored by decreasing the pillar relative spacing 


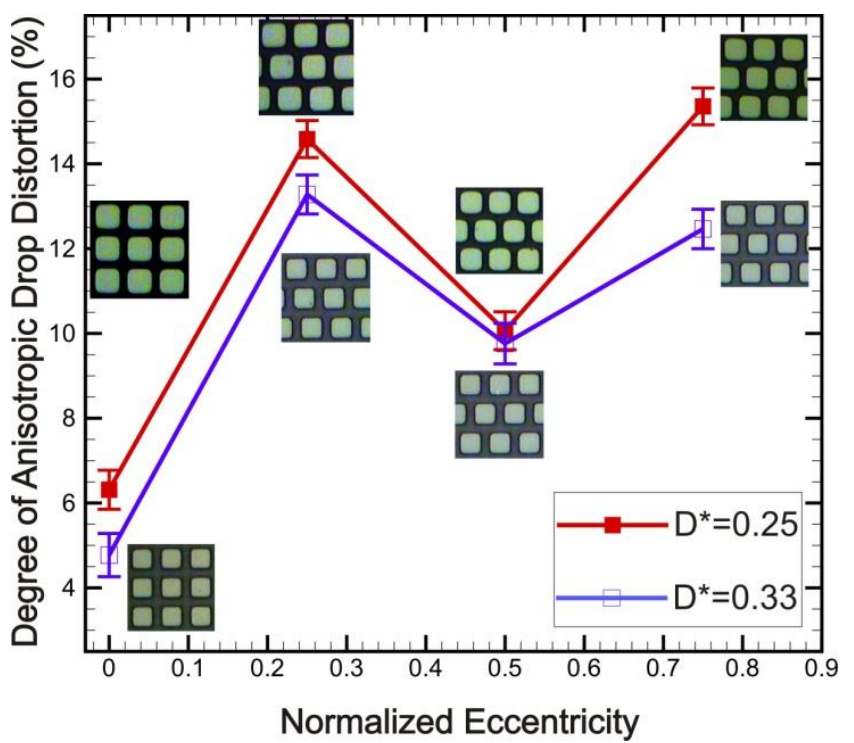

Fig. 5 Percentage of the degree of anisotropic droplet distortion $\zeta$ versus normalized eccentricity $\varepsilon^{*}$ at different values of relative pillar spacing $D^{*}$.

and increasing the pillar normalized eccentricity. This trend is 5 consistent with the contact angle anisotropy, which confirms the accuracy of the measurements. The maximum anisotropic droplet distortion $\zeta$ occurs for the case of $D^{*}=0.25$ and $\varepsilon^{*}=0.75$ where $\zeta=15 \%$ leads to $\delta=7 \%$. Whereas for the case of $D^{*}=0.33$ and $\varepsilon^{*}=0$, anisotropic droplet distortion $\zeta$ is less 10 than $5 \%$ and leads to an anisotropic wetting $\delta$ of $1.5 \%$. This fact explains the reason why top-view macroscopic images of the drop placed on the micropillar surfaces with zero eccentricities represent non-distorted, circular drop shape.

Furthermore, it should be noted that micropillars with $\varepsilon^{*}=$ $150.25,0.75$ are mirror images of each other. Thus, the morphologies of the pillars are identical. Consistently, the results in Figs. 4,5 confirm that the wetting anisotropy is similar on such surfaces, and droplets are elongated along the same mirror plane, i.e. y-axis in Fig. 1(a).

\section{${ }_{20}$ Three-phase Contact Line Shape on Anisotropic Pillars}

Owing to the existence of the roughness, the actual three-phase contact line of a droplet on rough surfaces is not smooth but rather corrugated which would give rise to the effect of contact line tension, an extra term in Gibbs free energy, as explained in 25 our previous paper ${ }^{35}$. This micro/nanoscale corrugation may be very small, thus, indistinguishable from smooth line macroscopically. For isotropic wetting/roughness, the shape of the contact line is assumed to be perfectly $\operatorname{circular}^{20}$. However, unequal droplet widths in contact with an isentropically rough 30 surface are key parameters in directional dependency of the contact angle. If the degree of anisotropy is high, such as surfaces with hydrophilic and hydrophobic strips as well as groove structures, the elongation of the drop along a specific direction is macroscopically observable. Chen et al. ${ }^{20}$ suggested an elliptical 35 geometry as the equivalent noncircular smooth contact line shape for the case of parallel groove structures as supported by their experimental data and numerical simulation.

The values of droplet aspect ratio $a^{*}$ are plotted in Figure 6 as a function of viewing angle $\alpha$ on a polar coordinate. To depict the 40 contact line shape of the droplet, spline interpolation is used to fit

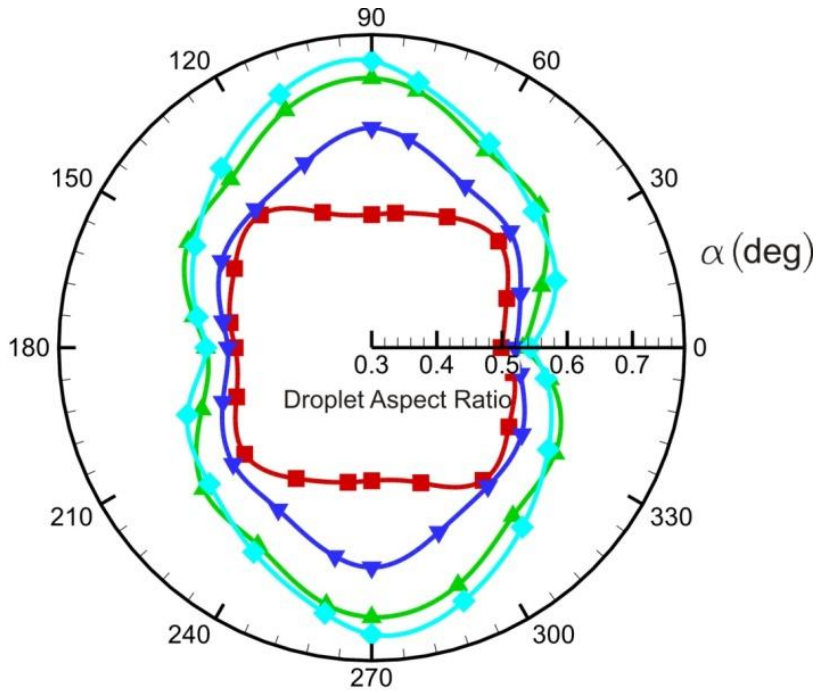

(a)

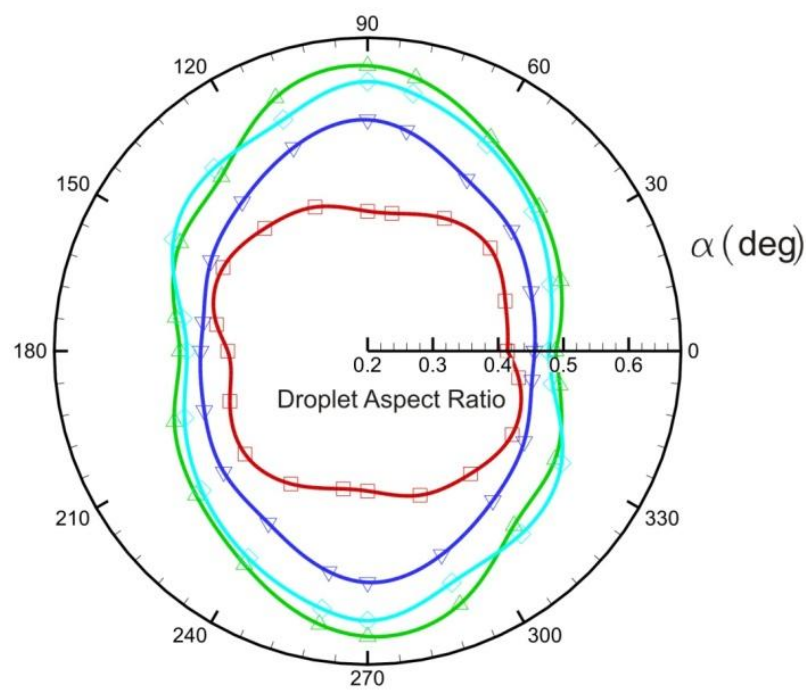

(b)

Fig. 6 Droplet aspect ratio $\boldsymbol{a}^{*}$, as a function of viewing angle $\boldsymbol{\alpha}\left(^{\circ}\right)$ on polar coordinate. Spline interpolation between the experimental date represents the three-phase contact line of the droplet (a) $\boldsymbol{D}^{*}=\mathbf{0 . 2 5}$; (b) ${ }_{45} \boldsymbol{D}^{*}=\mathbf{0 . 3 3}$ (line legend is the same as that of Fig. 3).

these experimental data. It should be noted that the values of aspect ratio for $\alpha=180^{\circ}-360^{\circ}$ are found based on the symmetrical conditions of the microstructures, i.e. the obtained curves from $\alpha=0^{\circ}-180^{\circ}$ are mirrored with respect to the 50 horizontal line. Fig. 6 clearly illustrates the corrugation of the three-phase contact line of the microstructured surfaces and its corresponding shapes by increasing the pillar eccentricity as well as the relative spacing. The result also illustrates that the variations of the contact line shape are more pronounced by 55 changing the micropillar eccentricities. That means wetting anisotropy is highly affected by the micropillar eccentricity, rather than relative spacing, as confirmed by the experimental contact angles shown in Fig. 3. 


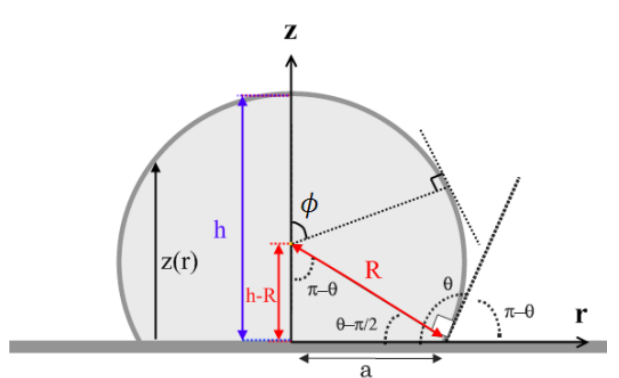

Fig. 7 Axisymmetric drop shape on a hydrophobic surface

\section{Semi-Analytical Model to Predict 3D Droplet Shape on Anisotropic Surface}

${ }_{5}$ To obtain the equilibrium drop shape on any surface, the Gibbs free energy of the surface should be minimized subject to some constraints such as constant drop volume condition forming the basis of numerical tools such as the Surface Evolver software ${ }^{41}$. However, for microstructured surfaces where the size of the 10 droplet is much larger than the characteristic length of the patterns, it was shown that finding the equilibrium drop shape is extremely difficult due to the computational complexity of the problem $^{20}$. Another alternative approach to find the equilibrium drop shape is to solve nonlinear partial differential equation of 15 Young-Laplace equation developed for the sessile drop. It can be proved mathematically that if the Bond number is less than unity, and the characteristic length of the drop is less than the capillary length $\delta_{c}$ of the liquid, the shape of the drop becomes spherical cap which is the solution of Young-Laplace equation by 20 neglecting the hydrostatic pressure. Such axisymmetric drop placed on a hydrophobic surface is illustrated in Fig. 7.

For an anisotropic surface at each viewing angle $\alpha$ the value of the contact angle $\theta$ is different. Thus, contact angle can be expressed as a function of $\alpha, \theta=f(\alpha)$.

${ }_{25}$ Using the current experimental data in Fig. 3, $f(\alpha)$ can be approximated as a Fourier series (with periods $T=\pi$ for nonzero eccentricities and $T=\pi / 2$ for zero eccentricities) as follows.

$\theta=f(\alpha)=a_{0}+\sum_{n=1}^{\infty} a_{n} \cos \frac{2 n \pi}{T} \alpha \approx a_{0}+a_{1} \cos \frac{2 \pi}{T} \alpha+$

$a_{2} \cos \frac{4 \pi}{T} \alpha$

30 where cosine Fourier series up to the first two sums are used for curve-fitting of the measured data. The values of $a_{0}, a_{1}$, and $a_{2}$ are listed in Table 2 for each micropillar surfaces.

Table 2 Coefficients of the Fourier series, Eq. (4), for the micropillars 35 used in this study.

\begin{tabular}{|c|c|c|c|c|c|c|c|c|}
\hline & \multicolumn{4}{|c|}{$D^{*}=0.25$} & \multicolumn{4}{|c|}{$D^{*}=0.33$} \\
\hline & $\begin{array}{c}\varepsilon^{*} \\
=0\end{array}$ & $\begin{array}{l}\varepsilon^{*} \\
=0.25\end{array}$ & $\begin{array}{l}\varepsilon^{*} \\
=0.50\end{array}$ & $\begin{array}{l}\varepsilon^{*} \\
=0.75\end{array}$ & $\begin{array}{l}\varepsilon^{*} \\
=0\end{array}$ & $\begin{array}{l}\varepsilon^{*} \\
=0.25\end{array}$ & $\begin{array}{l}\varepsilon^{*} \\
=0.50\end{array}$ & $\begin{array}{l}\varepsilon^{*} \\
=0.75\end{array}$ \\
\hline$a_{0}$ & 2.168 & 2.017 & 2.098 & 2.001 & 2.330 & 2.128 & 2.220 & 2.144 \\
\hline$a_{1}$ & 0.043 & 0.106 & 0.071 & 0.118 & 0.024 & 0.102 & 0.0719 & 0.091 \\
\hline$a_{2}$ & 0.000 & 0.012 & 0.001 & 0.000 & 0.001 & -0.009 & -0.009 & 0.009 \\
\hline
\end{tabular}

Using the geometrical relations as well as the fact that the height of the drop $h$ is constant at any viewing angle $\alpha$, the 2D form of the drop becomes:

40

$$
r(\phi)=\frac{h}{(1-\cos \theta)} \sin \phi
$$

$D^{*}=0.25 \quad D^{*}=0.33$ (a)

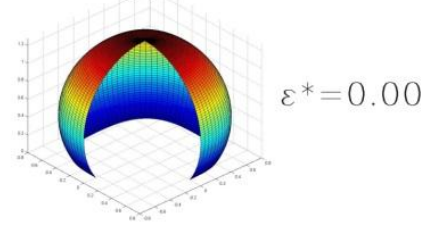

(b)

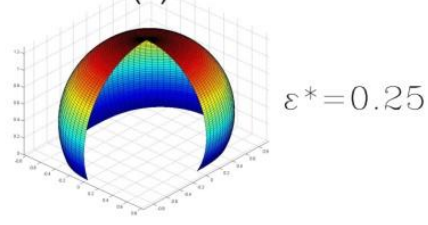

(c)

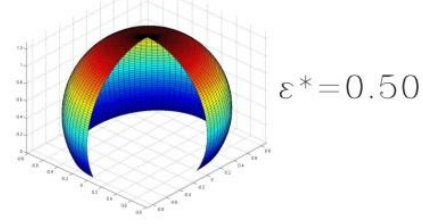

(d)

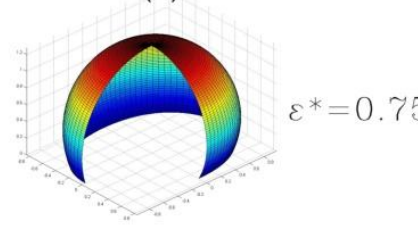

(e)

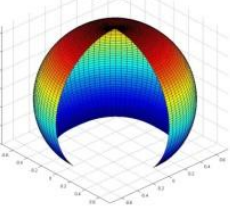

(f)

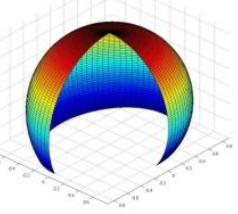

(g)

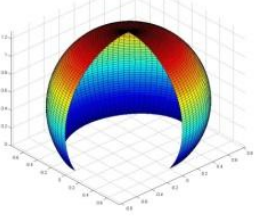

(h)

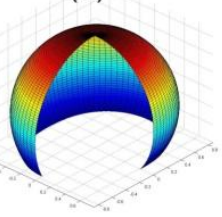

Fig. 8 Three-quarter of equilibrium drop shape with $V=2 \mu L$. From top to bottom, the effects of increasing the pillars eccentricity are shown, i.e. (a-d) and (e-h), while from left to right, the effects of increasing the 45 pillars relative spacing are illustrated, e.g. $(\mathrm{a}, \mathrm{e})$ or $(\mathrm{d}, \mathrm{h})$.

$$
z(\phi)=h-\frac{h}{(1-\cos \theta)}(1-\cos \phi)
$$

where $\phi$ is the zenith angle, as shown in Fig. 7. For an anisotropic surface at different viewing angles $\alpha$, the values of the contact angle $\theta$ are different. This continuous function was 50 obtained using Eq. (4). Therefore, the final 3D form of the equilibrium droplet shape on anisotropic surfaces becomes:

$$
\begin{aligned}
& x^{*}=\frac{\sin \phi}{1-\cos (f(\alpha))} \cos \alpha \\
& y^{*}=\frac{\sin \phi}{1-\cos (f(\alpha))} \sin \alpha \\
& z^{*}=1-\frac{1-\cos \phi}{1-\cos (f(\alpha))}
\end{aligned}
$$

where $0 \leq \phi \leq \theta, \quad 0 \leq \alpha \leq 2 \pi$, and $x^{*}=x / h ; y^{*}=y / h$; $z^{*}=z / h$.

Finally, by varying $\alpha$ from 0 to $360^{\circ}$, the 3D shape of the drop can be plotted. These shapes at different values of pillar relative 60 spacing and eccentricity are presented in Fig. 8(a-h). The results shown in Fig. 8 confirmed our previous discussions regarding the degrees of anisotropic wetting and droplet distortion by changing 
micropillars eccentricity and relative spacing. As illustrated in Fig. 8, increasing the relative pillar spacing $D^{*}$ as well as decreasing the pillar normalized eccentricity $\varepsilon^{*}$ make the droplet shape closer to the symmetrical configuration. If the geometric 5 parameters of the micropillars are tailored such that the anisotropic wetting increases, it can be concluded that the energy barriers of the surface also increases as compared to the isotropic features and wettability increases. This is in excellent agreement with the thermodynamic model presented by Zhao et al. ${ }^{23}$ derived

10 by calculating the wetting surface energy of the system as contact line moves along two orthogonal directions for parallel groove structures.

For the case of discrete micropillars, the obtained results can be explained by taking into consideration the corrugation of the 15 three-phase contact line which is the main reason of the directional dependency of the contact angle. Distorted nature of the three-phase contact line, as illustrated in Fig. 6, as well as corrugated drop shapes found from the simulated results (Fig. 8) by increasing anisotropic wetting, increases the value of the line 20 tension term of Gibbs free energy, i.e. the energy required to change the length of the three-phase contact line.

As explained in our previous paper ${ }^{35}$, the importance of the line tension becomes more pronounced when the contact line is corrugated. The corrugation gives rise to the energy barrier of the 25 system, and hence decreases the degree of hydrophobicity. For the case of zero eccentricity, i.e., Fig. 8(a,e) energy barrier is larger on the diagonal direction of the pillars, and as a result, the contact line would be distorted as shown in Fig. 6. Distortion of the contact line along the diagonal direction causes unequal drop 30 widths and leads to contact angle anisotropy. On the surfaces with nonzero eccentricities, line tension, equivalent to the energy barrier and contact line distortion, increases as the along $\alpha=90^{\circ}$ (y-axis in Fig. 1(a)). Likewise, as the sample rotates toward the features along $\mathrm{x}$-axis (orthogonal direction) energy barrier 35 decreases, and contact line corrugation becomes smaller and closer to the circular shape.

\section{Conclusions}

This paper experimentally investigated the anisotropic wetting of surfaces patterned with discrete micropillars. Square micropillars 40 with different values of relative spacing $D^{*}$ as well as normalized eccentricity $\varepsilon^{*}$, were fabricated using double casting PDMS replica molding. Contact angles were measured by gradually rotating the sample at different viewing angles $\alpha$. The contact angle anisotropy was found to be a periodic function of viewing

45 angle whose period was $\pi / 2$ and $\pi$ for zero and nonzero values of eccentricity, respectively. To quantify the directional dependency of the contact angle on such geometries both degrees of anisotropic wetting $\delta$ and droplet distortion $\zeta$ were defined. It was observed that both $\delta$ and $\zeta$ increased by increasing the pillar 50 eccentricity as well as decreasing the pillar relative spacing. However, the effect of changing the pillar eccentricity was much more pronounced. On the surface with $D^{*}=0.33$ and $\varepsilon^{*}=0$, minimum anisotropy was observed, i.e. $\delta_{\min }=1.5 \%$ and $\zeta_{\min }=4.8 \%$. By decreasing the relative pillar spacing to 0.25 55 and increasing the normalized eccentricity to 0.75 , maximum anisotropy was observed, i.e. $\delta_{\max }=7 \%$ and $\zeta_{\max }=15 \%$. Also, on the patterns which were mirror images of each other, $\varepsilon^{*}=0.25$ and $\varepsilon^{*}=0.75$, the contact angle anisotropy was consistently identical. Additionally, by measuring the droplet ${ }_{60}$ aspect ratio $a^{*}$ at each viewing angle, the three-phase contact line shape of the water droplet at each $D^{*}$ and $\varepsilon^{*}$ was found (2D approach). The three-phase contact line shapes on discrete anisotropic micropillars were highly corrugated at the microscopic scale. This fact explains why macroscopic images of 65 the drop placed on the micropillar surfaces with zero eccentricities represent non-distorted, circular drop shape. Furthermore, a semi-analytical model based on low Bond number, was developed to describe the equilibrium 3D shape of the droplet (3D approaches). The model is strictly based on the 70 relationship between droplet contact angle $\theta$ and viewing angle $\alpha$. This relationship was approximated by curve fitting of the experimental data using Fourier series. Based on the model, equilibrium 3D shapes of the droplet at different $D^{*}$ and $\varepsilon^{*}$ were found, and good qualitative agreement with the experimental 75 results was obtained. Both of these $2 \mathrm{D}$ and/or $3 \mathrm{D}$ approaches are extremely useful to detect the corrugated shapes of the threephase contact line. Generally, it was found that both wetting anisotropy of the micropillars and the degree of the hydrophobicity of the surface have the same trend and 80 interrelated. That means, by increasing micropillar normalized eccentricity, the energy barriers (fluctuations in the free energy) of the system also increase. This gives rise to the distortion of the three-phase contact line. The corrugation of the contact line is the main source of anisotropy. The results also showed that energy 85 barrier of the micropillar structures with nonzero eccentricities increases gradually in the orthogonal direction with a period of $180^{\circ}$, whereas for micropillars with zero eccentricities, it has a period of $90^{\circ}$. Finally, the results of the current paper suggested that combined effects of pillar relative spacing, normalized 90 eccentricity, and viewing angle should be taken into considerations when evaluating the contact angle anisotropy and wetting conditions of the microstructured surfaces.

\section{Appendix: Uncertainty and Error Propagation of the Experimental Data}

${ }_{95}$ Generally, if $z$ is a function of independent variables $x$ and $y$, $z=f(x, y)$, the uncertainty of $z, \Delta u_{z}$, becomes:

$$
\Delta u_{z}=\sqrt{\left(\frac{\partial f}{\partial x} \Delta u_{x}\right)^{2}+\left(\frac{\partial f}{\partial y} \Delta u_{y}\right)^{2}}
$$

100 where $\Delta u_{x}$ and $\Delta u_{y}$ are uncertainties of $x$ and $\mathrm{y}$, respectively. For $z=(x-y) /(x+y)$, it becomes:

$$
\frac{\partial f}{\partial x}=\frac{2 y}{(x+y)^{2}} \quad ; \quad \frac{\partial f}{\partial y}=\frac{-2 x}{(x+y)^{2}}
$$

Substituting Eq. (A2) in Eq. (A1) results:

$$
\Delta u_{z}=\frac{2}{(x+y)^{2}} \sqrt{y^{2} \Delta u_{x}{ }^{2}+x^{2} \Delta u_{y}{ }^{2}}
$$

105 This formula is used to calculate the uncertainties of $\delta$ by replacing $x \rightarrow \theta(\alpha)_{\max }$ and $y \rightarrow \theta(\alpha)_{\min }$ and are shown by error bars in Fig. 4. 
Similarly, the error propagation of $\zeta$ can be calculated by replacing $x \rightarrow a^{*}(\alpha)_{\max }$ and $y \rightarrow a^{*}(\alpha)_{\min }$ in Eq. (A3). To find the uncertainty of maximum/minimum droplet aspect ratio, $\Delta u_{a^{*} \max / \min }$, one can write:

${ }_{5} \Delta u_{a^{*} \max / \min }=\sqrt{\left(\frac{\Delta u_{a}}{h}\right)^{2}+\left(\frac{-a}{h^{2}} \Delta u_{h}\right)^{2}}=\frac{\Delta u_{a}}{h} \sqrt{1+a^{* 2}}$

where the uncertainties in $a$ and $h$ are calculated from:

$$
\Delta u_{h}=\Delta u_{a}=2 \sqrt{e_{b}^{2}+\left(\frac{e_{s}}{\sqrt{N}}\right)^{2}}
$$

In Eq. (A5), $e_{b}$ is the bias error as a result of the limited accuracy of the measuring the $a$ and $h, e_{b}=0.0005 \mathrm{~mm}$, and $e_{s}$ is the 10 systematic error due to deviation of the measured volume from the desired volume $(2 \mu L)$, and $N$ is the number of the samples. These uncertainties of $\zeta$ were represented by error bars in Fig. 5 .

\section{Notes and references}

${ }^{a}$ School of Mechanical and Aerospace Engineering, Nanyang 15 Technological University, Singapore 639798. Fax: 656791 1859; Tel: 65 6790 4457; E-mail: mntnguyen@ntu.edu.sg

1 L. Feng, S. Li, Y. Li, H. Li, L. Zhang, J. Zhai, Y. Song, B. Liu, L. Jiang and D. Zhu, Advanced Materials, 2002, 14, 1857-1860.

202 T. Sun, L. Feng, X. Gao and L. Jiang, Accounts of Chemical Research, 2005, 38, 644-652.

3 H. Wu, R. Zhang, Y. Sun, D. Lin, Z. Sun, W. Pan and P. Downs, Soft Matter, 2008, 4, 2429-2433.

4 Y. Zheng, X. Gao and L. Jiang, Soft Matter, 2007, 3, 178-182.

255 A. Neumann and R. Good, Journal of Colloid and Interface Science, 1972, 38, 341-358.

6 H. Gau, S. Herminghaus, P. Lenz and R. Lipowsky, Science, 1999, 283, 46-49.

7 S. Brandon, N. Haimovich, E. Yeger and A. Marmur, Journal of

$30 \quad$ Colloid and Interface Science, 2003, 263, 237-243.

8 M. Morita, T. Koga, H. Otsuka and A. Takahara, Langmuir, 2005, 21, 911-918.

9 O. Bliznyuk, E. Vereshchagina, E. S. Kooij and B. Poelsema, Physical Review E, 2009, 79, 041601-041606.

3510 H. P. Jansen, O. Bliznyuk, E. S. Kooij, B. Poelsema and H. J. W. Zandvliet, Langmuir, 2011, 28, 499-505.

11 E. Kooij, H. Jansen, O. Bliznyuk, B. Poelsema and H. Zandvliet, Colloids and Surfaces A: Physicochemical and Engineering Aspects, 2012, In Press.

4012 D. Zhu, X. Li, G. Zhang, X. Zhang, X. Zhang, T. Wang and B. Yang, Langmuir, 2010, 26, 14276-14283.

13 X. Zhang, Y. Cai and Y. Mi, Langmuir, 2011, 27, 9630-9637.

14 J. Y. Chung, J. P. Youngblood and C. M. Stafford, Soft Matter, 2007, 3, 1163-1169.

4515 C. Bukowsky, J. M. Torres and B. D. Vogt, Journal of Colloid and Interface Science, 2011, 354, 825-831.

16 A. Buguin, L. Talini and P. Silberzan, Applied Physics A: Materials Science \& Processing, 2002, 75, 207-212.

17 T. Wang, X. Li, J. Zhang, X. Wang, X. Zhang, X. Zhang, D. Zhu, Y. 50 Hao, Z. Ren and B. Yang, Langmuir, 2010, 26, 13715-13721.

18 T.-i. Kim and K. Y. Suh, Soft Matter, 2009, 5, 4131-4135.
19 H. Schonhorn, The Journal of Adhesion, 1987, 23, 147-161.

20 Y. Chen, B. He, J. Lee and N. A. Patankar, Journal of Colloid and Interface Science, 2005, 281, 458-464.

5521 A. D. Sommers and A. M. Jacobi, Journal of Micromechanics and Microengineering, 2006, 16, 1571-1578.

22 F. Zhang and H. Y. Low, Langmuir, 2007, 23, 7793-7798.

23 Y. Zhao, Q. Lu, M. Li and X. Li, Langmuir, 2007, 23, 6212-6217.

24 A. Sommers and A. Jacobi, Journal of Colloid and Interface Science, $60 \quad 2008, \mathbf{3 2 8}, 402-411$.

25 H. Kusumaatmaja, R. J. Vrancken, C. W. M. Bastiaansen and J. M. Yeomans, Langmuir, 2008, 24, 7299-7308.

26 W. Li, G. Fang, Y. Li and G. Qiao, The Journal of Physical Chemistry B, 2008, 112, 7234-7243.

6527 J. Yang, F. R. A. J. Rose, N. Gadegaard and M. R. Alexander, Langmuir, 2009, 25, 2567-2571.

28 L. Liu, A. M. Jacobi and D. Chvedov, Journal of Micromechanics and Microengineering, 2009, 19, 035026-035034.

29 C. J. Long, J. F. Schumacher and A. B. Brennan, Langmuir, 2009, 25, $70 \quad$ 12982-12989.

30 X. Yong and L. T. Zhang, Langmuir, 2009, 25, 5045-5053.

31 W. Choi, A. Tuteja, J. M. Mabry, R. E. Cohen and G. H. McKinley, Journal of Colloid and Interface Science, 2009, 339, 208-216.

32 D. Xia, X. He, Y. B. Jiang, G. P. Lopez and S. Brueck, Langmuir, $75 \quad 2010,26,2700-2706$.

33 D. Wu, Q. D. Chen, J. Yao, Y. C. Guan, J. N. Wang, L. G. Niu, H. H. Fang and H. B. Sun, Applied Physics Letters, 2010, 96, 053704-053706.

34 S. Neuhaus, N. D. Spencer and C. Padeste, ACS Applied Materials \& $80 \quad$ Interfaces, 2011, 4, 123-130.

35 N. Kashaninejad, W. K. Chan and N.-T. Nguyen, Langmuir, 2012, 28, 4793-4799.

36 D. Öner and T. J. McCarthy, Langmuir, 2000, 16, 7777-7782.

37 N. Kashaninejad, N.-T. Nguyen and W. K. Chan, Physics of Fluids

$85 \quad$ 2012, Under Review.

38 M. Sun, C. Luo, L. Xu, H. Ji, Q. Ouyang, D. Yu and Y. Chen, Langmuir, 2005, 21, 8978-8981.

39 A. Cassie and S. Baxter, Trans. Faraday Soc., 1944, 40, 546-551.

40 D. Quéré, Annu. Rev. Mater. Res., 2008, 38, 71-99.

9041 K. A. Brakke, Mathematics Department, Susquehanna Univerisity, Selinsgrove, PA, 2005, 1-233. 